\title{
Aberrant Carotid Artery Associated with Throat Discomfort and Retropharyngeal Mass
}

\author{
Yong Tae Hong ${ }^{1}$ (D) Sol Kim², Min Ji Kim², and Ki Hwan Hong' ${ }^{1}$ \\ 'Department of Otolaryngology-Head and Neck Surgery, Research Institute for Clinical Medicine of Jeonbuk National University, \\ Biomedical Research Institute of Jeonbuk National University Hospital, Jeonju, Korea \\ ${ }^{2}$ Department of Speech Pathology, Jeonbuk National University, Jeonbuk National University Hospital, Jeonju, Korea
}

\begin{abstract}
We present four cases of aberrant carotid arteries manifesting as pharyngeal masses with throat discomfort. The symptoms of this anomaly are very similar to those of laryngopharyngeal reflux or globus pharyngeus. The identification of this anomalous artery is essential for the head and neck surgeon because the anomaly frequently manifests as a symptomatic pulsatile mass in the pharynx. All patients complained of foreign body sensation in the throat and showed protrusion of the posterolateral pharyngeal wall anteromedially. CT scans demonstrated that there were two cases involving the right common carotid artery (CCA) and two cases involving both CCAs in the retropharyngeal space. As conclusion, the aberrant course of the artery can occur on both sides of CCAs, and it can manifest as a pharyngeal mass. When the patients complain of foreign body sensation in the throat without any other abnormal findings in the pharynx, we should consider the possibility that it may be due to the aberrant course of the CCA and its bifurcations in the retropharyngeal space.
\end{abstract}

Keywords Common carotid artery; Aberrant.

\section{INTRODUCTION}

The aberrant course of the carotid artery usually manifests no clinical signs and is discovered incidentally. The projection of an aberrant internal carotid artery almost always occurs in the lateral or posterolateral wall of the pharynx manifesting as a retropharyngeal mass. However, the projection of this aberrant artery is very rarely limited to the posterior pharyngeal wall. ${ }^{1-5)}$ This anomaly mostly occurs in the common carotid artery (CCA) and it is quite common and the female:male sex ratio is $3.3: 1$. In $71 \%$ of the cases, this anomaly was bilateral and $63 \%$ of anomalies occurred on the right side. ${ }^{2)}$

Although usually this anomaly is clinically silent, some patients can complain of throat discomfort such as foreign body sensation, chronic cough, mild dysphagia and dysphonia. ${ }^{2)}$ Most doctors tend to diagnose this anomaly as laryngopharyngeal reflux syndrome or globus pharyngeus. As already known, most patients with globus pharyngeus show no abnormality in the head and neck region. However, some patients show an aberrant course of the CCA which manifests as a retropharyngeal mass on contrasted computed tomography (CT) images of the neck. Clinically, we should be aware of two important things regarding aberrant carotid arteries. One is throat discomfort mimicking symptoms of laryngopharyngeal reflux disease (LPRD) and the other important thing is that it is mandatory to avoid catastrophic complications before head and neck surgery. These arteries are at risk for injury
Received February 13, 2020

Revised April 24, 2020

Accepted May 25, 2020

Corresponding Author

Ki Hwan Hong, MD, PhD

Department of Otolaryngology-

Head and Neck Surgery,

Jeonbuk National University,

Medical School,

20 Geonji-ro, Deokjin-gu,

Jeonju 54907, Korea

Tel +82-63-250-1990

Fax $+82-63-250-1986$

E-mail khhong@chonbuk.ac.kr

\section{ORCID iDs}

Yong Tae Hong (iD

https://orcid.org/0000-0001-7584-5823

Ki Hwan Hong (D)

https://orcid.org/0000-0003-2192-4838

This is an Open Access article distributed under the terms of the Creative Commons Attribution Non-Commercial License (https://creativecommons.org/ licenses/by-nc/4.0) which permits unrestricted non-commercial use, distribution, and reproduction in any medium, provided the original work is properly cited. 
during procedures frequently performed at the time of surgical interventions in the pharynx. In this report, we reviewed the aberrant transposition of the CCA and its bifurcations, internal and external carotid arteries, which manifested as a retropharyngeal mass.

\section{CASE REPORT}

We reviewed 4 cases of patients with aberrant carotid arteries. The first patient, a 62-year-old female, complained of foreign body sensation in her throat and a palpable mass in the thyroid region of the neck for 4 months. Her past history revealed that she had received drugs including proton pump inhibitor under the diagnosis of laryngopharyngeal reflux at a local clinic, but her symptoms did not improve at all. On physical examination, using fiberscopy, the right posterior pharyngeal wall was protruding forward (Fig. 1) and it was pulsating synchronously with the heartbeat. A hard fixed mass was palpated in the right thyroid area. No other abnormality was found in the head and neck region. She underwent ultrasound-guided fine needle aspiration for evaluating the thyroid mass. The cytologic results were reported as suspicious for papillary thyroid carcinoma. CT was performed. CT scans demonstrated that the right CCA showed an unusual medial course, initially displacing toward the midline at the level of the upper end of the thyroid cartilage. At the level of the hyoid bone and carotid sinus, the right CCA was located in the right retropharyngeal space and the left CCA was located normally on the lateral side of the hyoid bone (Fig. 2A). The right internal carotid artery (ICA) had an aberrant course, being situated medial to the external carotid artery (ECA) in the retropharyngeal space, but the left ICA was situated posterolateral to the ECA (Fig. 2B). For fur-
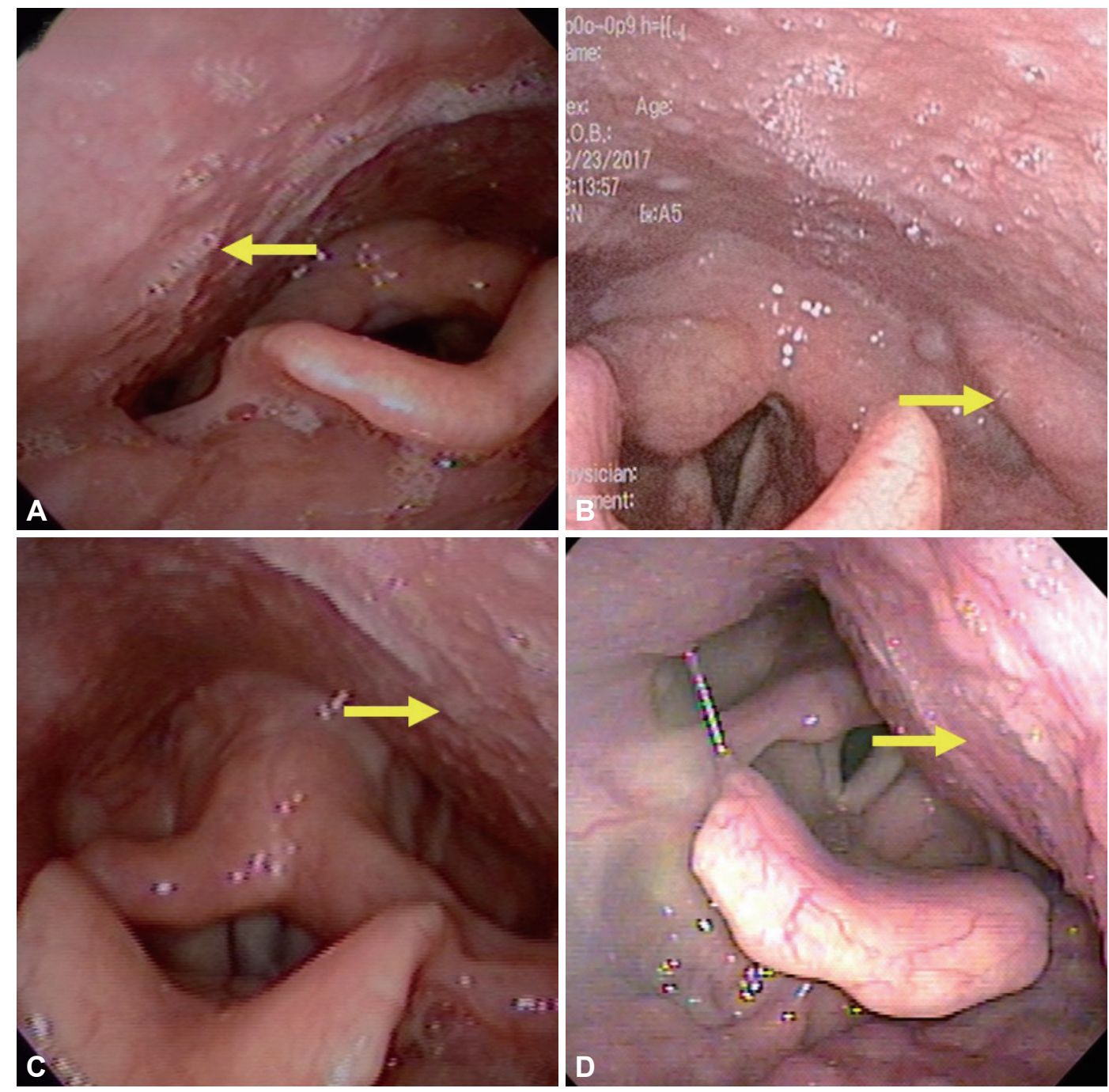

Fig. 1. Fiberscopic findings of the pharynx. The right oropharyngeal space showed narrowing due to protrusion of the aberrant carotid arteries (arrows). Right posterolateral protrusion of pharyngeal wall (A). Left lateral protrusion of carotid artery (B). Left posterolateral protrusion of pharyngeal wall $(C, D)$. 
ther evaluation, angiography was performed. Selective right CCA angiography demonstrated the aberrant course of the right CCA along the retropharyngeal space and marked medial deviation of the right ICA (Fig. 2C). Selective left CCA angiography showed a normal course and normal configuration (Fig. 2D). She received total thyroidectomy and central neck dissection and was discharged on the 5th day after surgery without any complications.

The other two female patients, 67 and 72 years old, complained of a lump-like sensation in their throat for more than 1 year. They were treated with medication under the impression of la- ryngopharyngeal reflux syndrome. However, their symptoms did not improve at all. On physical and endoscopic examinations for the 2nd case, there were no abnormal findings in the head and neck region. However, according to the endoscopic findings, both sides of the posterolateral pharyngeal wall were protruding and there was a mildly narrowed pharyngeal space, and it was pulsating synchronously with the heartbeat. CT scans demonstrated that the left CCA showed a retropharyngeal course. The internal carotid arteries coursed upward and more medial to the external carotid artery in the right retropharyn-
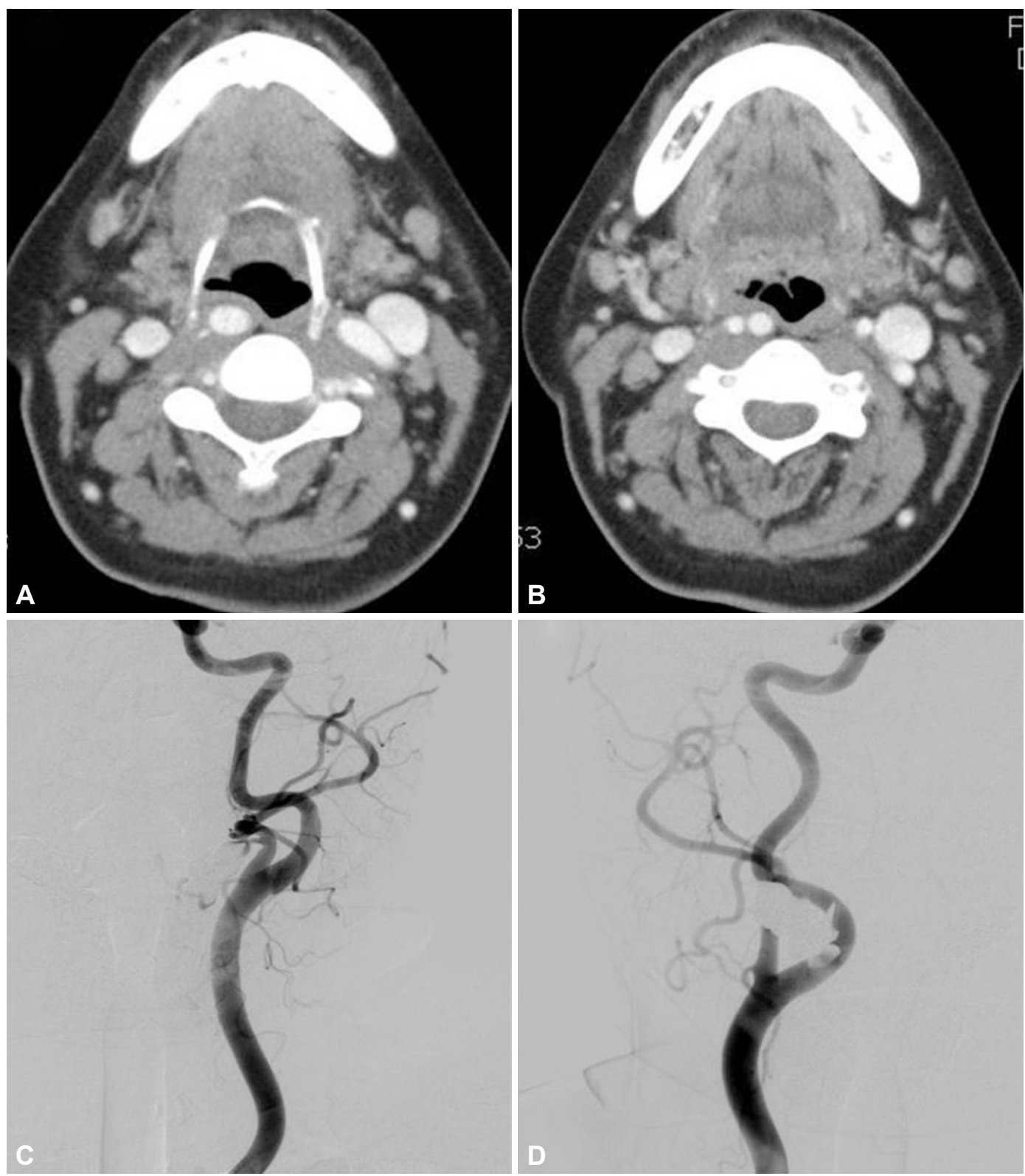

Fig. 2. Axial CT scan at the level of the hyoid bone and the carotid body (A). The right common carotid artery is located in the right retropharyngeal space. Axial CT scan above the hyoid bone (B). The right internal carotid artery is situated medial to the external carotid artery in the retropharyngeal space, but the left internal and external carotid arteries are situated normally. On bilateral carotid angiograms, the left internal carotid artery usually lies posterior and lateral to the external carotid artery $(C)$. However, the right internal carotid artery takes a course medial to the external carotid artery due to the course of the aberrant right internal carotid artery along the retropharyngeal space (D). 
geal space (Fig. 2A, B). On carotid angiograms, the left internal carotid artery usually lies posterior and lateral to the external carotid artery (Fig. 2C) and the right internal carotid artery takes a course medial to the external carotid artery (Fig. 2D).

In the 3rd case, both sides of the posterolateral pharyngeal wall were also mildly narrowed on endoscopic examination and were pulsating synchronously with the heartbeat. CT scans showed that both CCAs showed a retropharyngeal course (Fig. $3 \mathrm{~A}, \mathrm{~B})$. The right internal carotid arteries coursed upward and more medial to the external carotid artery in the right retropharyngeal space (Fig. 3C, D). The 4th patient, a 64-year-old male, also complained of foreign body sensation with mild dysphagia for 6 months. He also had been receiving medication for 6 months under the impression of laryngopharyngeal reflux syndrome, but his symptoms did not improve at all. On endoscopic examination, both sides of the posterior pharyngeal wall were protruding antero-medially and pulsating synchronously with the heart beat. CT scans demonstrated a large homogeneous mass in the left posterolateral pharyngeal space. The left carotid artery was deviated anterolaterally and the mass was suspicious of a benign neurogenic tumor (Fig. 4A). The right CCA showed a retropharyngeal course. The internal carotid artery coursed
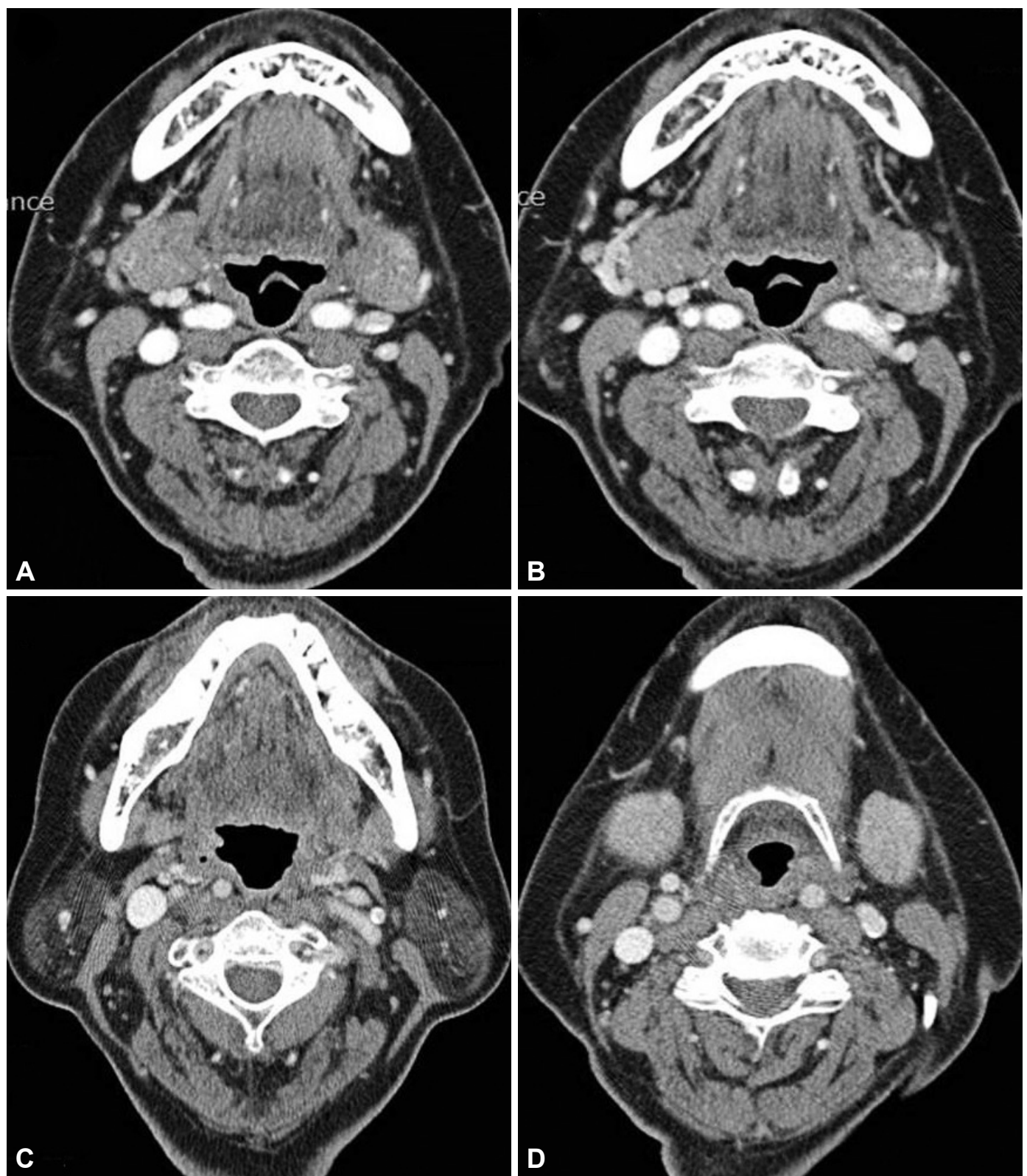

Fig. 3. Axial CT scan at the level of the epiglottic tip (A). Both common carotid arteries are located in the right retropharyngeal space. The right internal carotid artery is situated medial to the external carotid artery in the retropharyngeal space (B). Axial CT scan at the level of the mandibular body $(\mathrm{C})$. The right common carotid artery is located in the right retropharyngeal space. The left common carotid artery is situated in the retropharyngeal space at the level of low mandible (D). 
upward and more medially, nearly midline, to the external carotid artery (ECA) in the retropharyngeal space (Fig. 4B). We recommended surgical excision of the retropharyngeal mass, but the patient refused surgery and was lost to follow-up.

\section{DISCUSSION}

In recent years, LPRD has been attracting wide attention as a cause of not only globus sensation but also dysphonia and chronic cough. The reflux of gastric acid reaching the laryngopharynx is the basic pathological condition under the LPRD heading. LPRD is a collective term for conditions that present with unpleasant symptoms in the neck.

Various conditions can cause globus sensation in the throat and has focused on LPRD recently. Reflux of gastric acid is thought to cause globus sensation directly or indirectly, and these patients complain of unpleasant symptoms such as lumplike sensation, chronic cough, mild dysphonia and dysphagia. However, LPRD or globus phayngeus is often confused with other structural abnormalities of the head and neck region such as tumorous conditions or vascular anomalies. The aberrant course of the carotid artery is frequently found as a pulsatile mass on the lateral or posterolateral pharyngeal wall during routine physical examination of the head and neck region, but it is rarely limited to the posterior pharyngeal wall. Normally, the right CCA begins at the bifurcation of the brachiocephalic trunk. ${ }^{6}$ This CCA ascends within the carotid sheath with the internal jugular vein and the vagus nerve, called the carotid triangle. The CCA usually divides into to the internal and external carotid arteries at the level of the upper border of the thyroid cartilage or the hyoid bone, the C3-4 or C4-5 level. The external carotid artery can be distinguished easily from the internal carotid artery because it has several branches in the neck, and there are no branches of the internal carotid artery. ${ }^{7)}$ The cervical segment of the internal carotid artery can be further subdivided into a lower, sternomastoid portion and an upper, retrostyloid portion as it courses through the parapharyngeal space. The sternomastoid portion of the internal carotid artery is normally lateral to the pharyngeal wall, and posterior and slightly medial to the external carotid artery. The internal carotid artery enters the retrostyloid region after coursing above the inferior border of the posterior belly of the digastric muscle, and normally lies lateral to the pharyngeal wall. This artery approaches the skull base by summing a more medial location. ${ }^{8,9)}$

Leipzig and Dohrmann ${ }^{10)}$ divided the variations in the course of the carotid artery into the following 2 distinct categories: tortuosity and kinking. Elongation, redundancy, undulation, and an S-shaped curve are classified as tortuosity, while any sharp bend in the vessel is classified as kinking. This aberrant course may become more pronounced during adult life due to atherosclerotic or hypertensive changes in the wall of the artery. In contrast with the aberrant internal carotid artery, Fix et al. ${ }^{8)}$ reported an aberrant course of the CCA and its bifurcations (internal and external carotid arteries) in the posterior pharyngeal wall
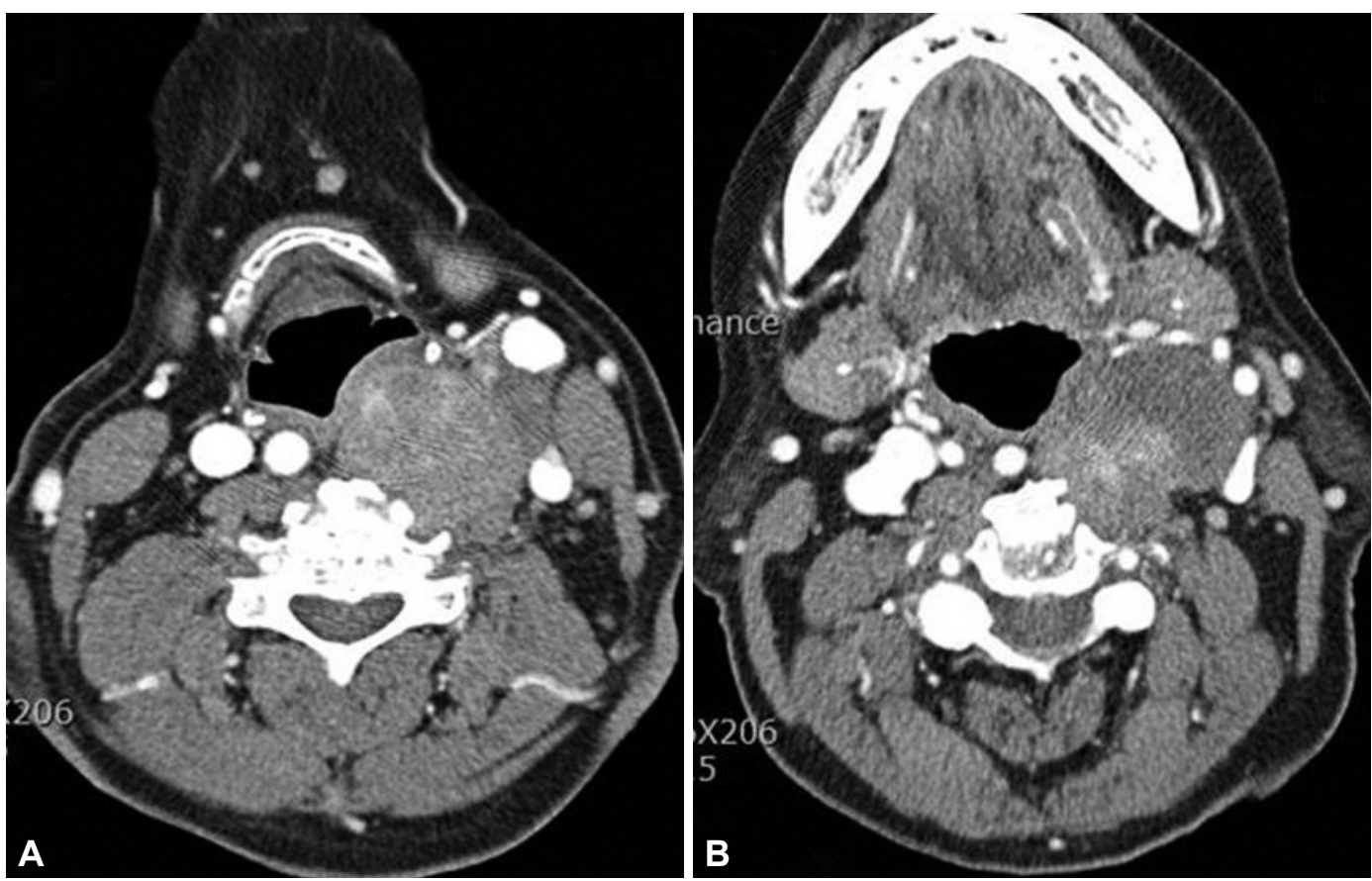

Fig. 4. Axial CT scan at the level of the hyoid bone (A). The right common carotid artery is located in the right retropharyngeal space. The right internal carotid artery is situated medial to the external carotid artery in the retropharyngeal space (B). A mixed echoic mass was noted in the left retro-lateral pharyngeal space and it was suspicious of a neurogenic tumor. 
and they described it as carotid transposition. Main causes of transposition of the CCA may be suspected to be atherosclerosis and hypertensive disease. ${ }^{11)}$

Awareness of the possibility of this unusual course of carotid arteries is important clinically. Arslan et al. ${ }^{12)}$ also reported that pulsatile mass detected on nasopharyngolaryngoscopy is most likely diagnosis as an aberrant CCA or cervical ICA. One possibility is throat discomfort related to symptoms with LPRD. Other possibility is that these arteries are at risk for injury during procedures frequently performed at the time of surgical interventions in the pharynx, especially in tonsillectomies. ${ }^{5)}$ In majority of cases, this anomaly is discovered only during routine oropharyngeal examination. Therefore, an appropriate examination of the pharynx is essential for proper diagnosis of patients with throat discomfort. However, as it is difficult to differentiated patients with aberrant carotid artery and LPRD in initial assesment, no further treatment was done. Other important thing is that it is mandatory to avoid catastrophic complications before head and neck surgery. Although angiography is still the gold standard for investigation of the status of the carotid arteries, CT and magnetic resonance (MR) provide essential information for the diagnosis of the aberrant course of the carotid artery. ${ }^{11)}$

In conclusion of this report, this anomaly should be essentially considered in patients with globus pharyngeus. Other important thing is to avoid catastrophic complications during pharyngeal or vascular surgery.

\section{Acknowledgments}

This paper was supported by Fund of Biomedical Research Institute, Chonbuk National University Hospital.

\section{Conflicts of Interest}

The authors have no financial conflicts of interest.

\section{Authors' Contribution}

Conceptualization: Yong Tae Hong. Data curation: Yong Tae Hong.
Formal analysis: Sol Kim. Methodology: Ki Hwan Hong. Project administration: Min Ji Kim. Resources: Ki Hwan Hong. Writing-original draft: Yong Tae Hong. Writing_review \& editing: Ki Hwan Hong. Approval of final manuscript: all authors.

\section{REFERENCES}

1. Hertzanu Y, Tovi F. Radiology case of the month. Aberrant internal carotid artery manifesting as a pharyngeal mass. J Otolaryngol 1992; 21(4):294-6

2. Schmäl F, Stoll W. Differential diagnosis and management of retropharyngeal space-occupying lesions. HNO 2002;50(5):418-23.

3. Miller S, Weill A, Maillard JC, Miaux Y, Chiras J. Aberrant internal carotid artery presenting in the midline retropharyngeal space: case report. Can Assoc Radiol J 1997;48(1):33-7.

4. Palacios E, Kirsch D, Rojas R. Anomalous course of the carotid arteries in the retropharyngeal space poses a surgical risk. Ear Nose Throat J 2005;84(6):336-7.

5. Ricciardelli E, Hillel AD, Schwartz AN. Aberrant carotid artery. Presentation in the near midline pharynx. Arch Otolaryngol Head Neck Surg 1989;115(4):519-22.

6. Osguthorpe JD, Adkins WY Jr, Putney FJ, Hungerford GD. Internal carotid artery as source of tonsillectomy and adenoidectomy hemorrhage. Otolaryngol Head Neck Surg 1981;89(5):758-62.

7. Paulsen F, Tillmann B, Christofides C, Richter W, Koebke J. Curving and looping of the internal carotid artery in relation to the pharynx: frequency, embryology and clinical implications. J Anat 2000;197 Pt 3(Pt 3):373-81.

8. Fix TJ, Daffner RH, Deeb ZL. Carotid transposition: another cause of wide retropharyngeal soft tissues. AJR Am J Roentgenol 1996;167(5): 1305-7.

9. Shanley DJ. Bilateral aberrant cervical internal carotid arteries. Neuroradiology 1992;35(1):55-6.

10. Leipzig TJ, Dohrmann GJ. The tortuous or kinked carotid artery: pathogenesis and clinical considerations. A historical review. Surg Neurol 1986;25(5):478-86.

11. Vega J, Gervas C, Vega-Hazas G, Barrera C, Biurrun C. Internal carotid artery transposition: another cause of widening of the retropharyngeal space. Eur Radiol 1999;9(2):347-8.

12. Arslan IB, Arslan Y, Demirhan E, Genc S, Pekcevik Y, Altin L, et al. Medially displaced common and internal carotid arteries presenting as a pulsatile mass: clinicoradiologic analysis of 62 cases. Ear Nose Throat J 2017;96(4-5):E1-7. 